\title{
FISIOTERAPIA NO PACIENTE RENAL CRÔNICO EM PROGRAMAÇÃO DE TRANSPLANTE DE RIM
}

\author{
Physical therapy in chronic renal patients in kidney transplant program
}

Debora Foger ${ }^{1,}$ Eloisa Aparecida Nelli ${ }^{2}$, Paulo Sérgio da Silva Santos ${ }^{1}$

\begin{abstract}
RESUMO
A equipe interdisciplinar é de extrema importância para o doente renal crônico, tanto durante o tratamento quanto pré e após a realização do transplante renal. Esta revisão de literatura teve como objetivo apresentar as disfunções nos sistemas respiratório, musculoesquelético e neurológico, oriundos do tratamento renal, bem como o impacto negativo na qualidade de vida dos pacientes e as propostas fisioterapêuticas para auxiliar na melhora da sobrevida desses indivíduos no preparo para o transplante de rim. Foi realizada uma busca nas bases de dados eletrônicas Scielo e PubMed, do ano de 2005 até a presente data, através dos descritores "insuficiência renal crônica", "fisioterapia", "diálise renal" e "equipe de cuidados de saúde", limitando a busca aos artigos publicados em inglês e em português, sem delimitações para tipos de estudos. O tratamento para doença renal crônica, bem como póstransplante, ocasionam efeitos negativos na saúde dos indivíduos, e a fisioterapia como parte integrante da equipe multidisciplinar ameniza os sintomas e melhora a qualidade de vida dos pacientes com doença renal crônica.
\end{abstract}

Descritores: Insuficiência Renal Crônica; Fisioterapia; Equipe de Cuidados de Saúde; Terapia Respiratória; Transplante de Rim.

Instituição:

1 Departamento de Cirurgia, Estomatologia Patologia e Radiologia - Centro de Pesquisa Clínica, Faculdade de Odontologia de Bauru Universidade de São Paulo. Bauru/SP, Brasil.

2 Centro de Pesquisas Clínicas - Hospital de Reabilitação de Anomalias Craniofaciais, Faculdade de Odontologia de Bauru Universidade de São Paulo. Bauru-SP, Brasil.

\section{Correspondência:}

Dr. Paulo Sérgio da Silva Santos

Faculdade de Odontologia de Bauru - Universidade de São Paulo Departamento de Cirurgia, Estomatologia Patologia e Radiologia Al. Dr. Otavio Pinheiro Brisolla 9-75 - CEP 17012-901 - Bauru/ SP Tel: (14) 3226-6113

E-mail: paulosergiosilvasantos@gmail.com

\section{INTRODUÇÃO}

No mundo, as doenças do rim e do trato urinário são responsáveis por aproximadamente 850 milhões de mortes anuais, e a incidência da doença renal crônica (DRC) aumenta em torno de $8 \%$ ao ano, sendo que, tanto a DRC quanto o seu tratamento (diálise e/ou hemodiálise) podem causar sérias disfunções em vários sistemas, como o muscular, ósseo, cardiovascular, metabólico e respiratório, diminuindo assim a qualidade da sobrevida dos pacientes. ${ }^{1,2}$

Atualmente, com a visão integral do ser humano, observa-se que cuidado em saúde não é mérito de um único profissional especialista, mas incumbência multiprofissional e interdisciplinar. ${ }^{3}$ Sendo assim, a fisioterapia através de seus diversos recursos 
terapêuticos pode auxiliar na melhora dos efeitos colaterais oriundos da terapia para o doente renal crônico, principalmente no que diz respeito aos sistemas respiratório e musculoesquelético, bem como na melhora da qualidade da sobrevida no pré e pós-transplante renal. Dessa forma, o presente estudo teve como objetivo levantar uma revisão de pesquisas destinadas à abordagem da fisioterapia em pacientes com doença renal crônica em planejamento de transplante de rim.

\section{MATERIAL E MÉTODOS}

Realizamos uma revisão da literatura a partir do ano de 2005 até o presente, em livros e nas bases eletrônicas de dados "Scielo" e "PubMed", através dos descritores, "insuficiência renal crônica", "fisioterapia”, "diálise renal" e "equipe de cuidados de saúde", limitando a busca aos artigos publicados em inglês e em português, estudos com seres humanos, população alvo com indivíduos adultos, sem delimitações para tipos de estudos. A revisão foi ampliada através de outros estudos citados para fundamentação e discussão do tema. Assim, a literatura selecionada foi organizada de forma a elucidar a definição de doença renal crônica, os efeitos que essa patologia causa nos sistemas respiratório, musculoesquelético, neurológico e a abordagem fisioterapêutica na referida área.

\section{DOENÇA RENAL CRÔNICA}

A DRC é um distúrbio patológico, progressivo e irreversível das funções glomerular, tubular e endócrina do rim, apresentando uma taxa de filtração glomerular (TFG) menor que $60 \mathrm{ml} / \mathrm{min} / 1,73 \mathrm{~m} 2$, por um período superior a três meses. Quando o nível de TFG é menor que $15 \mathrm{ml} / \mathrm{min} / 1.73 \mathrm{~m} 2$, a doença renal crônica passa a ser denominada insuficiência renal crônica na fase terminal (ICRT) ou dialítica. ${ }^{4-7}$ Sendo o rim um órgão responsável pela manutenção da homeostase, equilíbrio hídrico ácido básico e eletrolítico, e por participar de funções hormonais e regulação da pressão arterial, o paciente renal crônico necessita de tratamentos como a hemodiálise (HD) ou a diálise peritoneal (DP), que substituirão parcialmente a função dos rins, enquanto se espera por uma solução definitiva, que é o caso do transplante renal. ${ }^{1,2,6,8,9}$

A origem da insuficiência renal crônica envolve doenças primárias dos rins, doenças sistêmicas e doenças hereditárias. ${ }^{2}$ Suas causas mais comuns são: diabetes mellitus, glomerulonefrites, nefro esclerose hipertensiva, doença renovascular, rins policísticos, uropatias obstrutivas e malformações congênitas, entretanto, as situações mais importantes são o diabetes mellitus e a hipertensão arterial sistêmica, conjuntamente com o histórico familiar de doença renal, levando esse indivíduo a apresentar maior prevalência da necessidade de terapia renal substitutiva, que por sua vez reduz os sintomas da doença, não sendo, porém, uma terapia curativa. ${ }^{8,10-13}$ Com a danificação da função renal, os demais órgãos e sistemas são envolvidos e começam a atuar de maneira anormal, onde só há chances de sobrevivência do paciente através da diálise ou do transplante renal. ${ }^{7}$

Na rotina clínica, a nefrologia não inclui prevenção para deterioração da função física, para que essa área da medicina vá além da dosagem da diálise e avaliações laboratoriais, passando a incluir o estudo do declínio funcional é necessária a avaliação efetiva da função física desses pacientes. ${ }^{14} \mathrm{O}$ tipo de teste utilizado para avaliar a função física, dependerá do objetivo da avaliação, das características da população de interesse, como idade, comorbidades, status ambulatorial, função cognitiva e níveis de atividade física. Devem também ser consideradas necessidades operacionais, incluindo pessoal especializado, tempo de avaliação para os testes, local (clinica ou laboratório) e a cronometragem de teste em relação ao tratamento de diálise (para considerar fatores como fadiga, balanço, fraqueza), o peso do paciente, entre outros. ${ }^{15}$

Algumas possibilidades de avaliação da função física são o prejuízo fisiológico, que frequentemente resulta em limitações de performance física, requer avaliação em laboratório, com equipamentos especializados e técnicos treinados, a avaliação de limitação de performance física que é determinado utilizando alguns testes de performance física, realizado ambulatorialmente e a avaliação de incapacidade ou participação ativa que é tipicamente determinado utilizando autorrelato. ${ }^{15,16}$

Alguns tipos de mensuração de avaliação fisiológica são as de aptidão cardiorrespiratória (máxima absorção de oxigênio, pico de absorção de oxigênio, testes de exercícios submáximos), e as de função muscular (força, energia, resistência). Já as mensurações de mobilidade e capacidade de desempenho, são os testes ambulatoriais (caminhada de seis minutos, teste de caminhada intermitente, velocidade de marcha, teste de potência de subir escada, sentar na cadeira repetidamente, tempo para levantar e ir, teste de andar e escalar), e o teste de autorrelato (índice de Katz para atividades de vida diária, índice de Lawton para atividades de vida diária e instrumental, Escala de função física SF-36). Avaliar a função física dos pacientes com doença renal crônica é de extrema importância para identificar indivíduos que se beneficiarão de intervenções preventivas, pacientes 
com alto risco de morte precoce, para monitorar e identificar declínios de função que podem indicar um novo problema de saúde, estratificar riscos para intervenções clínicas complexas e monitorar os efeitos das intervenções clínicas e ambientais, como por exemplo, a atividade física. ${ }^{15-17}$

\section{A DOENÇA RENAL E O SISTEMA RESPIRATÓRIO}

O sistema respiratório é afetado negativamente pela doença renal e pelo seu tratamento, ${ }^{2,4}$ sendo que algumas mudanças na função muscular respiratória, na mecânica pulmonar e nas trocas gasosas ocorrem com frequência nesses pacientes. A disfunção pulmonar no paciente com doença renal crônica pode ser um resultado considerado direto, ou seja, devido à circulação das toxinas uremias, ou indireto, através da sobrecarga do volume, anemia, supressão imunológica, calcificação óssea, desnutrição, desordens eletrolíticas e desequilíbrio ácido-base..$^{3,9}$

A perda da capacidade respiratória nesses pacientes ocorre principalmente devido à diminuição da força dos músculos respiratórios; essa diminuição dos volumes e capacidades respiratórias causa dificuldade e, em alguns casos, incapacidade de execução de exercícios. ${ }^{2}$

Outras complicações também são encontradas no tecido pulmonar dos pacientes com insuficiência renal crônica; são elas: edema pulmonar, derrame pleural, fibrose e calcificação pulmonar e pleural, hipertensão pulmonar, diminuição do fluxo sanguíneo capilar pulmonar e hipoxemia. Pode ocorrer também carência no fornecimento de oxigênio para os músculos, devido à diminuição da microcirculação periférica, diminuição da síntese de ATP muscular por deficiências na utilização de carboidratos, resistência à insulina, alterações das enzimas glicoliticas e diminuição da oxidação dos ácidos graxos. 2,9

O tratamento hemodialítico prejudica a função pulmonar devido à uremia, sendo que esse quadro pode ser acentuado por uma doença pulmonar latente. A literatura mostra que o tratamento hemolítico afeta de qualquer maneira a função e força muscular respiratória, porém, esse comprometimento do sistema respiratório ocorre de forma progressiva e está ligado ao maior período de tempo e à quantidade de sessões de hemodiálise realizadas. ${ }^{2,9}$

\section{A DOENÇA RENAL, O SISTEMA MUSCULO- ESQUELÉTICO E A QUALIDADE DE VIDA}

Qualidade de vida relacionada à saúde é um conceito muito complexo e nos dias de hoje não apresenta uma perfeita definição, porém inclui aspectos físicos, psicológicos e sociais que podem ser expressos de várias formas, de acordo com a percepção subjetiva dos indivíduos. ${ }^{18}$

A literatura mostra que existe um impacto negativo na qualidade de vida dos portadores de insuficiência renal crônica que são submetidos ao tratamento hemodiálico trazendo sérias limitações físicas, psicológicas e sociais. ${ }^{4,8}$ A obrigatoriedade do tratamento é um aspecto importante em relação ao impacto na qualidade de vida dos pacientes, principalmente pela diminuição da capacidade física, perdas biopsicossociais como o desemprego, degradação da imagem corporal, restrições dietéticas, hábitos sedentários, e, por consequência disso, aumento da mortalidade entre esses indivíduos. . $^{4,10}$

Há diversos fatores que se relacionam com a qualidade de vida do paciente com doença renal crônica, sendo que as alterações musculoesqueléticas estão entre as mais importantes. Sabe-se que o rim é um órgão que participa ativamente do ajuste da homeostase mineral; sendo assim, a insuficiência renal pode gerar alterações no esqueleto. ${ }^{19}$ As doenças ósseas resultantes da DRC são chamadas de osteodistrofia renal; além dessa, há algumas manifestações atribuídas à DRC e diálise, como a deposição de alumínio, deposição de amiloide, espondiloartropatia destrutiva, ruptura de tendão, deposição de cristal, infecção e necrose avascular, entre outras. ${ }^{15} \mathrm{Em}$ geral, a artralgia é muito comum em indivíduos que foram submetidos à dialise, uma vez que $70 \%$ deles relatam sintomas articulares que tendem a ser mais prevalentes, de acordo com a duração do tratamento. Os pacientes, normalmente, também se queixam de rigidez matinal, principalmente na articulação do joelho, porém, manifestações clínicas e radiológicas possuem maior prevalência após dez anos de diálise, como é o caso da síndrome do túnel do carpo, dor articular inflamatória, cistos ósseos e espodiloartropatias. As irregularidades osteoarticulares em pacientes portadores de insuficiência renal crônica submetidos a tratamento de diálise manifestam-se com frequência; dessa forma, é de extrema importância diagnosticar e realizar o devido tratamento de maneira precoce, com o intuito de amenizar a sintomatologia dolorosa, melhorar a capacidade funcional e, consequentemente, proporcionar melhor qualidade de vida a esses pacientes. ${ }^{16-20}$

O sistema muscular também é afetado no paciente com insuficiência renal crônica, principalmente devido à diminuição da ingestão protéico-calórica, atrofia muscular por desuso e desbalanço protéico muscular, prejudicando de maneira rigorosa as fibras musculares tipo II, causando diminuição do leito vascular e capilar, 
ocorrência de calcificação intravascular e redução do fluxo sanguíneo local. ${ }^{2,5,9}$

São frequentes as queixas de pacientes portadores de DRC sobre desconforto e/ou dor nas regiões lombar e cervical; isso ocorre principalmente devido à presença de distúrbios musculoesqueléticos, à permanência na posição sentada durante o tratamento e má postura do paciente nas cadeiras padronizadas para diálise, que em alguns casos não respeitam as individualidades ergonômicas de cada indivíduo. É de extrema importância a realização de exercícios de fortalecimento para indivíduos sob hemodiálise, visando a melhora da realização de suas atividades cotidianas. ${ }^{3,8,21}$

A fraqueza muscular generalizada também é um sintoma que ocorre nesses pacientes; geralmente, os membros inferiores e a musculatura proximal são mais prejudicados. A etiologia desse sintoma é multifatorial, porém, sua principal causa é a deficiência de vitamina D. Outras causas associadas podem ser: hiperparatireoidismo, intoxicação por alumínio, neuropatia periférica, toxidade por drogas como, por exemplo, os corticosteroides, alterações nas concentrações de cálcio e fósforo e calcificação vascular, provocando isquemia. A falta de atividade física também é associada à patogênese da fraqueza muscular; além disso, a transferência prejudicada de oxigênio para o músculo pode ser uma causa de miopatia urêmica; nesses casos, consegue-se a melhora da miopatia através de exercícios progressivos de resistência. ${ }^{19}$

As complicações cardiovasculares também causam impacto muito negativo na qualidade de vida do paciente com DRC submetido à HD, pois contribuem para a baixa tolerância ao exercício, e de maneira consequente, para a dificuldade na realização das atividades básicas e instrumentais da vida diária. O processo de HD é uma condição que beneficia o sedentarismo nos pacientes renais crônicos, sendo que este apresenta grande influência negativa para o sistema cardiovascular e capacidade funcional, além de estar profundamente ligado à mortalidade na $\mathrm{DRC} .^{20-22} \mathrm{O}$ tratamento dialítico nos pacientes com DRC está associado a restrições funcionais, bem como a diversas complicações físicas que comprometem a qualidade de vida desses pacientes. O transplante renal é uma boa alternativa de tratamento em longo prazo, porém tem sido dada pouca atenção às estratégias para melhorar a qualidade de vida dos pacientes que realizaram esse tipo de tratamento. ${ }^{18}$

\section{FISIOTERAPIA PARA O DOENTE RENAL E SEU IMPACTO NA QUALIDADE DE VIDA}

A qualidade de vida dos doentes crônicos tem se mostrado um critério de extrema importância na classificação da efetividade de tratamentos na área da saúde. Assim, a literatura mostra que a inclusão de um programa de fisioterapia pode trazer benefícios às alterações físicas e psíquicas que ocorrem pela introdução da diálise no cotidiano dos pacientes renais crônicos (Figura 1).$^{10} \mathrm{~A}$ realização de atividade física tem efeito positivo na saúde mental e emocional, obtendose grande melhora nos níveis de depressão, ansiedade, sono, humor, controle de peso, liberação de emoções e frustrações, que são características dos pacientes portadores de DRC. Um programa de fisioterapia durante o período hemolítico motiva o paciente em um ambiente estruturado e monótono, além de melhorar as dimensões de capacidade funcional, dor, vitalidade, saúde mental, atividade de vida diária e trabalho, apesar de todos os sintomas frequentes nesse tipo de paciente, como cansaço, fadiga, desânimo, entre outros. ${ }^{8,20}$

Figura 1: Principais alterações encontradas na DRN e estratégias fisioterapêuticas para minimizá-las.

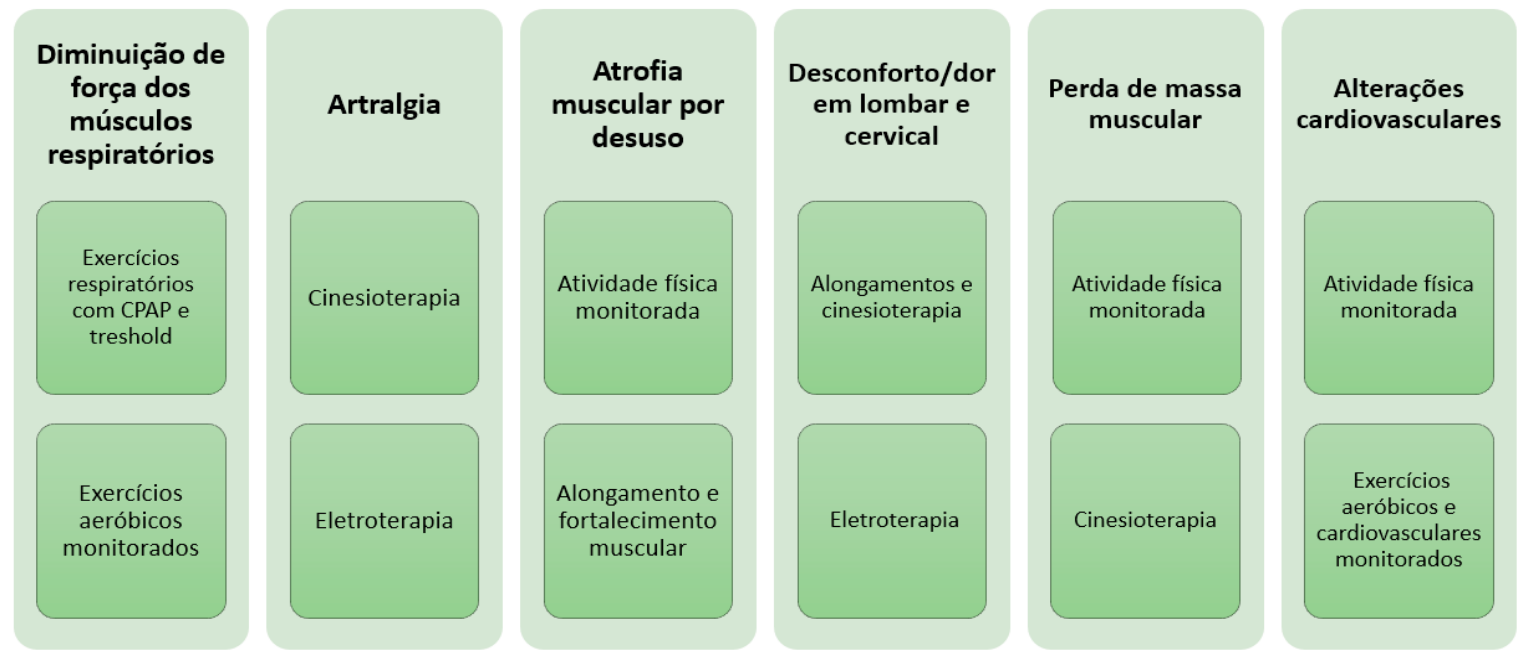


A literatura demonstra que programas de exercícios aeróbicos intradialíticos podem causar muitos benefícios. Para o impacto negativo que o tratamento hemodiálitico causa ao sistema respiratório, a fisioterapia pode auxiliar através de intervenções, como a realização de exercícios respiratórios através do uso de pressão positiva contínua na via aérea (CPAP), treinamento de força muscular respiratória com a manovacuometria e o uso do treshold, juntamente com a associação de exercícios aeróbicos utilizando bicicleta ergométrica e/ ou esteira. .,9,22 $^{2}$

Os exercícios físicos nos pacientes que passam pelo tratamento de hemodiálise são bastante divulgados em centros nos EUA e na Europa, porém, infelizmente no Brasil, poucos locais adotam essa prática na rotina dos pacientes. ${ }^{22}$

Existem algumas contraindicações de exercícios durante a diálise; são elas: queixas álgicas, dispneia, níveis pressóricos elevados, hipotensão hidradialítica, ganho excessivo de peso no período interdialítico e dificuldade no acesso vascular. ${ }^{22}$

Sabe-se que a população de renais crônicos apresenta aumento dos índices de óbito por doenças cardiovasculares; dessa forma, é de extrema importância o estimulo da atividade física nessa população, com o intuito de reduzir drasticamente esses eventos cardiovasculares, e consequentemente, melhorar a qualidade de vida dos indivíduos renais crônicos. ${ }^{5,9,19}$ Portanto, a fisioterapia pode auxiliar, elaborando um programa de exercícios com intensidade, frequência e duração, adequado às realidades de cada serviço e de cada paciente, respeitando seus limites, idade e capacidade física. ${ }^{20}$

Estudo com cinquenta pacientes objetivou avaliar um programa de fisioterapia realizado em pacientes com doença renal crônica durante hemodiálise. As avaliações foram realizadas antes e 16 meses após o início do treinamento, consistindo de exercícios de fortalecimento muscular, alongamento e bicicleta ergométrica estacionária. As análises foram compostas pelo teste de caminhada de seis minutos, nível de esforço de BORG, teste de repetição máxima (força em quadríceps), qualidade de vida e medidas de pressão arterial, frequências cardíaca e respiratória. Como resultado, foram encontrados nesse estudo aumento na distância percorrida e da força de quadríceps, redução da frequência cardíaca e respiratória, melhora do score total da qualidade de vida, mas, principalmente no que diz respeito à capacidade funcional. Sendo assim, o estudo concluiu que a fisioterapia com base em um programa de exercícios durante a hemodiálise pode proporcionar melhora significativa da qualidade de vida e capacidade física em pacientes renais crônicos. ${ }^{23} \mathrm{~A}$ análise de vinte e dois estudos realizada através de uma revisão sistemática mostrou que a perda de massa muscular é o mais significante preditor de mortalidade nos pacientes em hemodiálise, e o exercício físico é um importante fator no controle e reversão da perda muscular. Os estudos dessa revisão demonstraram algumas limitações metodológicas como tamanho da amostra e falta de homogeneidade dos exercícios. Porém, mesmo assim, os autores concluíram que programas de exercícios físicos adequadamente prescritos durante a hemodiálise são seguros para os pacientes e podem gerar benefícios; no entanto, esses programas durante hemodiálise ainda não se tornaram rotina na maioria dos centros dialíticos, provavelmente pelas lacunas deixadas na literatura, bem como pela escassez de profissionais qualificados para implementação de tais programas. ${ }^{24}$

Apesar da importância da atividade física durante a terapia dialítica e na vida diária dos pacientes com doença renal crônica, é importante salientar alguns cuidados que devem ser tomados antes de iniciar qualquer atividade física e durante a mesma. O paciente renal crônico deve evitar realizar exercícios físicos quando apresentar febre (acima de $38,3^{\circ} \mathrm{C}$ ), quando perder uma sessão de diálise, caso apresente nova doença ainda não tratada, ou quando o exercício causar dor. Existem alguns sintomas que quando presentes no paciente renal crônico durante a atividade física há necessidade de interromper a atividade; são eles: falta de ar, dores ou pressão no tórax, batimentos cardíacos irregulares, náusea, cãibras nas pernas, tontura, dor ou pressão no pescoço ou na mandíbula, excesso de fadiga e borrão na visão. É extremamente necessário que o paciente consulte um médico antes de começar a se exercitar novamente..$^{25}$

\section{OUTRAS ALTERAÇÕES CLÍNICAS DE PACIENTES RENAIS CRÔNICOS EM HEMODIÁLISE}

Pacientes submetidos à hemodiálise também apresentam sintomas físicos e emocionais, sendo que os mais frequentes são: insônia, síndrome das pernas inquietas, prurido urêmico e transtornos neurológicos. Diversos estudos com grande número de participantes têm demonstrado que pelo menos metade de todos os indivíduos avaliados apresenta distúrbios do sono, dor, doença arterial coronariana, distúrbios psiquiátricos, sendo que todos esses sintomas foram relacionados ao mau sono. ${ }^{26}$

\section{- Distúrbios do sono}

Para pacientes que apresentam distúrbio do sono, o 
tratamento deve iniciar com higiene do sono, ou seja, revisão e modificação dos rituais de sono, modificação do ambiente, identificação de qualquer uso de cafeína ou estimulante antes do sono, além da terapia comportamental cognitiva para insônia. Caso após a higiene do sono o paciente não apresente resultados satisfatórios, indica-se tratamento farmacológico. ${ }^{26}$

Um estudo foi realizado com 40 pacientes com doença renal crônica submetidos à hemodiálise. Foram utilizados dois instrumentos, o Diário do sono para avaliar a quantidade de sono e o índice de qualidade de sono de Pittsburg para avaliar a qualidade do sono dos participantes. Como resultado, a maioria dos pacientes do estudo (80\%) apresentou quantidade de sono abaixo do satisfatório e qualidade de sono ruim ou distúrbios do sono. ${ }^{27}$

A fisioterapia pode de certa forma auxiliar na melhora do sono desses pacientes através da massoterapia, que são técnicas terapêuticas de mobilização dos tecidos superficiais e profundos, causando relaxamento da musculatura. ${ }^{28}$ Apesar de ser um tema ainda pouco evidenciado na literatura, um estudo demonstrou melhora da qualidade do sono em pacientes em diálise que receberam massagem com acupressão durante 12 minutos, três vezes por semana, durante o período de tratamento em diálise, comparando-se com controles que não receberam tratamento ou receberam acupressão simulada. ${ }^{29}$

\section{- Síndrome das pernas inquietas}

A síndrome das pernas inquietas é um transtorno sensório-motor neurológico com prevalência de 12\% a $25 \%$ em pacientes com doença renal crônica sob diálise. Essa síndrome é caracterizada pela sensação de um impulso incontrolável de mover as pernas. Esses impulsos começam ou pioram em períodos de inatividade, e são parcial ou completamente aliviados com o movimento. Ocorrem predominantemente à noite ou em períodos de repouso. ${ }^{30} \mathrm{~A}$ doença está ligada a histórico familiar; alguns poucos estudos demonstram que apesar da patologia ser incerta, a doença renal pode desempenhar um papel nessa síndrome, já que a prevalência da mesma diminui em pacientes que realizaram transplante renal. ${ }^{31} \mathrm{~A}$ base de evidência para tratamento dessa síndrome é pequena; estudos randomizados mostram que 0 tratamento pode ser dividido em abordagens farmacológicas e não farmacológicas, sendo que a primeira categoria de tratamento farmacológico é composta de medicamentos que influenciam as vias de dopamina. ${ }^{32,33}$ As sessões de diálise duram aproximadamente quatro horas e, como durante os períodos de inatividade os sintomas são exacerbados, o momento do tratamento dialítico apresenta risco aumentado para que esses sintomas ocorram. Sendo assim, quanto ao tratamento não farmacológico, alguns pequenos estudos mostraram que o exercício aeróbico durante as sessões de diálise podem diminuir a gravidade dos sintomas. ${ }^{34-36}$

A fisioterapia pode auxiliar na melhora dos sintomas dessa síndrome através da inclusão de um programa de exercícios aeróbicos durante a hemodiálise. O treinamento físico e tratamento com agonistas de dopamina (DA) têm sido utilizados com sucesso para a melhora dos sintomas da síndrome das pernas inquietas (SPI) em pacientes em hemodiálise. ${ }^{37} \mathrm{Um}$ estudo randomizado duplo-cego investigou os efeitos do treinamento de exercícios intradialíticos durante seis meses, em combinação com uma dose baixa de DA em pacientes que sofrem sintomas da síndrome das pernas inquietas. Quatorze pacientes estáveis com SPI em hemodiálise foram distribuídos aleatoriamente para o grupo de treinamento de exercícios associado à DA e para o grupo de treinamento de exercícios associado ao placebo. Ambas as combinações demonstraram reduzir igualmente os sintomas da SPI em $60 \%$; sendo assim, pôde-se concluir que a dose de DA com treinamento de exercícios aeróbicos pode ser considerada uma abordagem alternativa a dosagens elevadas de DA na redução da gravidade dos sintomas de SPI nesses pacientes. ${ }^{37}$

\section{- Prurido Urêmico}

O prurido urêmico é um sintoma comum nos pacientes com doença renal crônica, e está associado à diminuição da qualidade de vida e depressão. ${ }^{38}$ Para esse tipo de sintoma, existe o tratamento tópico para escamação da pele, com a realização de hidratação adequada através do uso de creme emoliente aquoso duas a quatro vezes ao dia. O tratamento sistêmico atualmente mais utilizado para o prurido urêmico é a gabapentina. Quando existe intolerância à gabapentina, o uso de pregabalina nas doses de 25 a $75 \mathrm{mg}$ à noite é indicado. Outros tratamentos como a fototerapia, e acupuntura também são indicados. ${ }^{39,40}$

A Fisioterapia pode auxiliar no tratamento não farmacológico do prurido urêmico, através da aromaterapia, fototerapia e acupuntura, quando o profissional é devidamente especializado nessas áreas. Um estudo com 29 pacientes com doença renal crônica submetidos à hemodiálise avaliou os efeitos da aromaterapia no prurido; treze pacientes receberam massagem de aromaterapia no braço, três vezes por semana, durante quatro semanas. Antes e após o tratamento, foram avaliados o índice de prurido, o $\mathrm{pH}$ da pele, a hidratação do estrato córneo e os marcadores bioquímicos relacionados ao prurido. Os resultados 
mostraram que, após a aromaterapia, o índice de prurido foi significativamente diminuído, $\mathrm{opH}$ da pele não apresentou alterações e a hidratação do estrato córneo aumentou. Dessa forma, os resultados desse estudo mostraram que o uso de aromaterapia pode ser um método útil e eficaz de tratamento de prurido em pacientes submetidos à hemodiálise. ${ }^{41}$

A acupuntura é utilizada para tratamento de várias condições, mas seus efeitos sobre o prurido urêmico em pacientes com doença renal crônica não são totalmente claros. Uma Revisão sistemática teve como objetivo avaliar a eficácia da acupuntura para o prurido urêmico em pacientes com doença renal crônica. Três ensaios clínicos randomizados e três estudos observacionais não controlados foram incluídos na pesquisa. Todos os ensaios incluídos relataram os efeitos benéficos da acupuntura. No entanto, a maioria dos estudos mostrou alto risco de viés. Dessa forma, a evidência encontrada através dessa revisão sistemática foi insuficiente para mostrar que a acupuntura é um tratamento eficaz para prurido urêmico em pacientes com doença renal crônica em hemodiálise principalmente pela falta de rigor metodológico dos estudos incluídos. ${ }^{42}$

\section{- Disfunções neurológicas}

Pacientes com doença renal geralmente apresentam múltiplas disfunções orgânicas causadas, em parte, pela comunicação entre o rim e outros órgãos e tecidos. As anormalidades da função cognitiva e os altos níveis de incidência de depressão são características de pacientes com hemodiálise. ${ }^{43}$ Existe interferência de substâncias entre o rim e o cérebro, indicado pela apresentação frequente de transtornos neurológicos como doença cerebrovascular, comprometimento cognitivo e neuropatia durante doença renal crônica. ${ }^{44,45}$ $\mathrm{O}$ advento dos programas de diálise e transplante renal levou a uma redução na taxa de complicações neurológicas associadas à uremia, porém, um novo conjunto de complicações surgiu como consequência dos efeitos da diálise no sistema nervoso central em curto e longo prazo. ${ }^{46}$

Os pacientes com DRC apresentam prevalência cinco vezes maior de doença cerebrovascular clínica e subclínica, sintomas neurológicos mais graves e pior resultado clínico geral, quando comparados aos que não possuem. Como rim e cérebro estão expostos ao fluxo sanguíneo de alto volume, são susceptíveis a danos vasculares e patologias microvasculares. Estudo demonstrou que existe prevalência aumentada de lesões de substância branca em pacientes portadores de DRC. As lesões de matéria branca representam o acúmulo de células degenerativas na matéria branca do cérebro e podem causar acidente vascular encefálico (AVE), demência e morte. A incidência anual de AVE é de $10 \%$ em pacientes portadores de DRC, sendo que existem alguns fatores de risco convencionais para ocorrência do AVE em pacientes em diálise, como idade avançada, hipertensão e diabetes mellitus; alguns fatores de risco não convencionais são anemia, estresse, desnutrição e inflamação. ${ }^{47-51}$

A diálise pode causar alguns efeitos agudos nos indivíduos, como a síndrome de desequilíbrio de diálise, uma condição que normalmente ocorre ao final de uma sessão de diálise. É uma consequência extrema do comprometimento cognitivo associado à diálise, onde os pacientes podem sofrer alguns sintomas como dor de cabeça, náusea e confusão durante ou após o tratamento.

Anormalidades neurológicas são comumente encontradas em pacientes com DRC; sendo assim, são sugeridos testes de avaliação desses pacientes, como o Minimental e o Montreal, que devem ser realizados com regularidade. ${ }^{52,53}$

A interação entre o rim e o cérebro é um processo complexo, que pode manifestar-se em numerosas patologias. Tal como ocorre interferência renal com outros órgãos, é essencial que as comorbidades neurológicas sejam efetivamente gerenciadas e/ou prevenidas e os mecanismos subjacentes elucidados para facilitar o desenho de intervenções apropriadas para retardar o curso da doença renal. É, portanto, necessária maior conscientização de comprometimento cognitivo em pacientes com doença renal. ${ }^{46}$

A Fisioterapia, em suas diversas áreas de atuação, também tem papel importante na Neurologia, pois atua ativamente nas doenças que acometem o Sistema Nervoso Central ou Periférico que levam a distúrbios neurológicos, motores e cognitivos. No caso dos pacientes com doença renal crônica que apresentam sintomas neurológicos, o fisioterapeuta é capaz de avaliar os déficits funcionais e cognitivos, e a partir daí traçar um plano de tratamento mais adequado individualizado para cada paciente, visando, por exemplo, melhora da força, coordenação, dos padrões motores, do equilíbrio e demais necessidades do paciente que precisam ser reestabelecidas. ${ }^{28}$

Como visto anteriormente, a incidência anual de AVE em pacientes com doença renal crônica é de 10\%; sendo assim, será relatada a atuação da fisioterapia nessa patologia.

Os principais comprometimentos diretos do AVE são: défficits somatossensitivos, visuais, mo ᄀtores, dor, alterações no tônus, padrões sinergísticos anormais, 
reflexos anormais, paresia, distúrbios de controle da postura e equilíbrio, distúrbios da fala e lingua $g e m$, disfagia, disfunção perceptiva, disfunção cognitiva, distúrbios afetivos, diferenças comportamentais entre os hemisférios, crises e disfunção da bexiga e do intestino. $^{54}$ A trombose venosa profunda (TVP) e o embolis $\neg$ mo pulmonar são complicações que podem ocorrer em todos os pacientes que permaneçam imobilizados, porém, para o AVE, a incidência da TVP é de $47 \%$, e $10 \%$ dos óbitos que ocorrem nesses pacientes são por embolia pulmonar. A hemiparesia e as disfunções motoras presentes nesses pacientes resultam em dificuldade para execução de movimentos, o que consequentemente piora a qualidade de vida pela falta de independência principalmente nas atividades de vida diária e instrumentais. ${ }^{54,55}$

A recuperação após acidente vascular encefálico deve ser o mais precoce possível, pois a melhora funcional é mais rápida durante os primeiros meses após o AVE, melhorando assim, o prognóstico da doença. No entanto, a fisioterapia é extremamente necessária para que os ganhos funcionais aconteçam desde o diagnóstico do AVE até durante muitos anos. A conduta fisioterapêutica tem como principal objetivo nesses pacientes maximizar a capacidade funcional, evitando assim complicações secundárias. ${ }^{56}$

Atualmente, os recursos fisioterapêuticos têm cada vez mais aprofundado-se na ciência e no aperfeiçoamento. Os principais recursos utilizados para a reabilitação dessa população são a cinesioterapia, hidroterapia, hipnoterapia, massoterapia, termoterapia, eletroterapia e técnicas alternativas, ou seja, exercícios terapêuticos não convencionais. ${ }^{57} \mathrm{O}$ fisioterapeuta é capaz de identificar e avaliar a melhor estratégia de reabilitação do paciente em suas diversas fases e o prognóstico do paciente dependerá de múltiplas variantes, incluindo fatores sociais e psicológicos que afetam o paciente com AVE. ${ }^{28}$

\section{CONSIDERAÇÕES FINAIS}

Os sintomas musculoesqueléticos, respiratórios e neurológicos presentes em pacientes renais crônicos são bastante comuns, principalmente quando o tratamento dialítico torna-se longo. Manifestações como ombro doloroso, síndrome do túnel do carpo, dedos em gatilho, fraqueza muscular, câimbras, hipoxemia, síndrome das pernas inquietas, alterações do sono, prurido urêmico e diminuição da função pulmonar reforçam que a abordagem fisioterapêutica nesses pacientes é essencial tanto para minimização das sequelas como para reabilitação das complicações físicas, funcionais e psicossociais, precoces ou tardias, decorrentes dos tratamentos. Considerando a complexidade da doença e a escassez de material científico sobre a abordagem da fisioterapia em pacientes renais, inclusive sobre as recomendações do manuseio desses pacientes quanto à existência de limitações cardio-pulmonares e de mobilidade, investigações adicionais serão necessárias, a fim de aumentar a abrangência das informações disponíveis sobre técnicas fisioterapêuticas e orientar tal prática relacionada a indivíduos com DRC.

\section{ABSTRACT}

The interdisciplinary team is extremely important for the chronic renal patient, both during treatment and after renal transplantation. This literature review had as objective to present the dysfunctions in the respiratory, musculoskeletal and neurological systems from the renal treatment as well as the negative impact on the quality of life of the patients and the physiotherapeutic proposals to help improve the survival of these individuals in the preparation for kidney transplantation. A search was made in the electronic databases Scielo and PubMed, from 2005 to the present, using the descriptors "chronic renal failure", "physiotherapy", "renal dialysis" and "patient care team", limiting the search for articles published in English and Portuguese, without delimitations for types of studies. Treatment for chronic kidney disease as well as post-transplant causes negative effects on the health of individuals, and physiotherapy as an integral part of the multidisciplinary team alleviates the symptoms and improves the quality of life of patients with chronic kidney disease.

Keywords: Renal Insufficiency, Chronic; Physical Therapy Modalities; Patient Care Team; Respiratory Therapy; Kidney Transplantation. 


\section{REFERÊNCIAS}

1-Siviero PCL, Machado CJ, Cherchiglia ML. Chronic kidney failure by means of multiple causes of death in Brazil. Cad Saúde Colet. 2014;22(1):75-85.

2-Menezes Junior CAV, Guia MJ, Perão KA, Santos E. Repercussões da doença renal crônica e da hemodiálise na função pulmonar: uma revisão bibliográfica. UNILUS Ensino Pesq. 2013;10(20):21-34.

3-Diniz DP, Carvalhaes JTA. Equipes multiprofissionais em unidades de diálise: contribuição ao estudo da realidade brasileira. J Bras Nefrol. 2002;24(2):88-96.

4-Jatobá CJP, Amaro WF, Andrade APA, Cardoso FPF, Monteiro AMH, Oliveira MAM. Avaliação da função pulmonar, força muscular respiratória e teste de caminhada de seis minutos em pacientes portadores de doença renal crônica em hemodiálise. J Bras Nefrol. 2008;30(4):280-7.

5-Souza AC, Albuquerque PR, Queiroz TBN. Avaliação da força dos músculos ventilatórios e condicionamento físico em pacientes portadores de insuficiência renal crônica submetidos à hemodiálise. Rev Insp Mov Saúde. 2012;4(21):1-5.

6-Soares KTA, Viesser MV, Rzniski TAB, Brum EP. Eficácia de um protocolo de exercícios físicos em pacientes com insuficiência renal crônica, durante o tratamento de hemodiálise avaliada pelo SF-36. Fisioter Mov. 2011;24(1):133-40.

7-Freire APC, Rios CS, Moura RSM, Burneiko RCVM, Padulla SAT, Lopes FS. Aplicação de exercício isotônico durante a hemodiálise melhora a eficiência dialítica. Fisioter Mov. 2013;26(1):167-74.

8-Lara CR, Santos FAOG, Silva TJ, Camelier FWR. Qualidade de vida de pacientes renais crônicos submetidos à fisioterapia na hemodiálise. Ciênc Saúde. 2013;6(3):163-71.

9-Cury JL, Brunetto AF, Aydos RD. Efeitos negativos da insuficiência renal crônica sobre a função pulmonar e a capacidade funcional. Rev Bras Fisioter. 2010;14(2):91-8.

10-Padulla SAT, Matta MV, Melatto T, Miranda RCV, Camargo MR. A fisioterapia pode influenciar na qualidade de vida de indivíduos em hemodiálise. Cienc Cuid Saude. 2011;10(3):564-70.

11-Lienert RSC, Figueiredo CEP, Figueiredo AEPL. Evolução do peso durante o primeiro ano de transplante renal e a ocorrência de Diabetes Mellitus após 5 anos de seguimento. Ciênc Saúde. 2014;7(3):148-54.

12-Tzvetanov IP, West-Thielke G, D’Amico M, Johnsen A, Ladik G, Hachaj M, et al. A Novel and Personalized Rehabilitation Program for Obese Kidney Transplant Recipients. Transplant Proc. 2014;46:3431-7.

13-Lôbo MCSG, Bello VAO. Reabilitação Profissional Pós Transplante Renal. J Bras Nefrol. 2007;29(1):29-32.

14-Painter P, Marcus RL. Assessing physical function and physical activity in patients with CKD. Clin J Am Soc Nephrol. 2013;8:861-72.
15-Koufaki P, Mercer T: Assessment and monitoring of physical function for people with CKD. Adv Chronic Kidney Dis. 2009;16:410-19.

16-Koufaki P, Kouidi E: Current best evidence recommendations on measurement and interpretation of physical function in patients with chronic kidney disease. Sports Med. 2010;40:1055-1074.

17-Painter PL, Stewart AL, Carey S: Physical functioning: Definitions, measurement, and expectations. Adv Ren Replace Ther. 1999;6:110-23.

18-Vieira WP, Gomes KWP, Frota NB, Andrade JECB, Vieira RMRA, Moura FEA, et al. Manifestações musculoesqueléticas em pacientes submetidos à hemodiálise. Rev Bras Reumatol. 2005;45(6):357-64.

19-Kastelz A, Tzvetanov IG, Fernhall B, Shetty A, Gallon $L$, West-Thielke $P$, et al. Experimental protocol of a randomized controlled clinical trial investigating the effects of personalized exercise rehabilitation on kidney transplant recipients' outcomes. Contemp Clin Trials. 2015;45:170-6.

20-Reboredo MM, Faria RS, Portes LH, Mol CG, Pinheiro BV, Paula RB. Exercício aeróbico durante a hemodiálise: relato de cinco anos de experiência. Fisioter Mov. 2011;24(2): 239-46.

21-Greenwood SA, Koufaki P, Mercer THB, Rush R, O'Connor $E$,Tuffnell $R$, et al. Aerobic or resistance training and pulse wave velocity in kidney transplant recipients: a 12-week pilot randomized controlled trial (the exercise in renal transplant [exert] trial). Am J Kidney Dis. 2015;66(4):689-98.

22-Teplana V, Mahrovab A, Pithac J, Racekd J, Gürliche J, Teplan V, et al. Early Exercise Training After Renal Transplantation and Asymmetric Dimethylarginine: The Effect of Obesity. Kidney Blood Press Res. 2014;39:289-98.

23-Silva SF, Pereira AA, Silva, WAH, Simôes R, Neto JRB. Physical therapy during hemodialyse in patients with chronic kidney disease. J Bras Nefrol. 2013;35(3):170-6.

24-Coelho DM, Ribeiro JM, Soares DD. Physical Exercise During Hemodialysis: A Systematic Review. J Bras Nefrol. 2008;30(2):88-98.

25-Painter P. A Guide for People on Dialysis. Medical Education Institute, Inc.2000.

26-Lindner AV, Novak M, Bohra M, Mucsi I. Insomnia in patients with chronic kidney disease. Semin Nephrol. 2015;35:359-72.

27-Guimarães CKD, Alves DAG, Guimarães LHCT. Evaluation of the quality and quantity of sleep in chronic renal failure patients on hemodialysis. Rev Neurocienc. 2011;19(4):609-13.

28-Piassaroli CAP, Almeida GC, José Carlos LuvizottoJC, Suzan ABBM. Physical Therapy Rehabilitation Models in Adult Patients with Ischemic Stroke Sequel. Rev Neurocienc. 2012;20(1):128-37.

29-Markell MS. Potential benefits of complementary medicine modalities in patients with chronic kidney disease. Adv Chronic Kidney Dis. 2005;12(3):292-9. 
30-Novak M, Winkelman JW, Unruh M. Restless legs syndrome in patients with chronic kidney disease. Semin Nephrol. 2015;35:347-58.

31-Venkateshiah SB, loachimescu OC. Restless legs syndrome. Crit Care Clin. 2015;31:459-72.

32-Trenkwalder C, Stiasny K, Pollmacher T, et al. I-Dopa therapy of uremic and idiopathic restless legs syndrome: a doubleblind, crossover trial. Sleep. 1995;18:681-88.

33-Walker SL, Fine A, Kryger MH. I-DOPA/carbidopa for nocturnal movement disorders in uremia. Sleep. 1996;19:214-18.

34-Giannaki CD, Sakkas GK, Hadjigeorgiou GM, et al. Nonpharmacological management of periodic limb movements during hemodialysis session in patients with uremic restless legs syndrome. ASAIO J. 2010;56:538-42.

35-Giannaki CD, Hadjigeorgiou GM, Karatzaferi C, et al.A single-blind randomized controlled trial to evaluate the effect of 6 months of progressive aerobic exercise training in patients with uraemic restless legs syndrome. Nephrol Dial Transplant. 2013;28:2834-40.

36-Mortazavi M, Vahdatpour B, Ghasempour A, et al. Aerobic exercise improves signs of restless leg syndrome in end stage renal disease patients suffering chronic hemodialysis. Sci World J. 2013;2013:628142.

37-Giannaki CD, Sakkas GK, zaferi CK, ki MDM, kis, YK, Hadjigeorgiou GM, nidis LNS. Combination of exercise training and dopamine agonists in patients with RLS on dialysis: a randomized, double-blind placebo-controlled study. ASAIO J. 2015;61(6):738-41.

38-Pisoni RL, Wikstrom B, Elder SJ, et al. Pruritus in haemodialysis patients: international results from the Dialysis Outcomes and Practice Patterns Study (DOPPS). Nephrol Dial Transplant. 2006;21:3495-505.

39-Aperis G, Paliouras C, Zervos A, Arvanitis A, Alivanis P. The use of pregabalin in the treatment of uraemic pruritus in haemodialysis patients. J Renal Care. 2010;36:180-5.

40-Solak Y, Biyik Z, Atalay H, et al. Pregabalin versus gabapentin in the treatment of neuropathic pruritus in maintenance haemodialysis patients: a prospective, crossover study. Nephrology (Carlton). 2012;17:710-17.

41-RoYJ, HaHC, KimCG, YeomHÁ.The effects of aromatherapy on pruritus in patients undergoing hemodialysis. Dermatol Nurs. 2002;14(4):231-4.

42-Kim KH1, Lee MS, Choi SM. Acupuncture for treating uremic pruritusin patients with end-stage renal disease: a systematic review. J Pain Symptom Manage. 2010;40(1):117-25.

43-McIntyre CW, Goldsmith DJ. Ischemic brain injury in hemodialysis patients: which is more dangerous, hypertension or intradialytic hypotension? Kidney Int. 2015;87(6):1109-15.
44-National Kidney Foundation. KIDOQI clinical practice guidelines for chronic kidney disease: evaluation, classification, and stratification. Am. J. Kidney Dis. 2002;39:S1-266.

45-Tilki, H. E., Akpolat, T., Cos,kun, M. \& Stålberg, E. Clinical and electrophysiologic findings in dialysis patients. J. Electromyogr. Kinesiol.2009; 19:500-8.

46-Lu R, Kiernan MC, Murray A, Rosner MH, Ronco C. Kidney-brain crosstalk in the acute and chronic setting. Nat Rev Nephrol. 2015;11(12):707-19

47-Wardlaw, J. M., Sandercock, P. A., Dennis, M. S. \& Starr, $\mathrm{J}$. Is breakdown of the blood-brain barrier responsible for lacunar stroke, leukoaraiosis, and dementia? Stroke.2003; 34: 806-12.

48-Seliger, S. L. et al. Risk factors for incident stroke among patients with end-stage renal disease. J. Am. Soc. Nephrol.2003;14:2623-31.

49-United States Renal Data System 2006 Annual Report. Morbidity \& mortality. Neuroepidemiology: Incident \& prevalent stroke [online] http://www.usrds.org/2006/pdf/06_ morb_morte_06.pdf (2006).

50-Seliger, S. L. \& Longstreth, W. T. Jr. Lessons about brain vascular disease from another pulsating organ, the kidney. Stroke. 2008;39:5-6.

51-Wetmore, J. B. et al. Atrial fibrillation and risk of stroke in dialysis patients. Ann. Epidemiol. 2013;23:112-8.

52-Nasreddine, Z. S. et al. The Montreal Cognitive Assessment, MoCA: a brief screening tool for mild cognitive impairment. J. Am. Geriatr. Soc. 2005;53:695-9.

53-Tariq, S. H. et al. Comparison of the Saint Louis University mental status examination and the minimental state examination for detecting dementia and mild neurocognitive disorder-a pilot study. Am. J. Geriatr. Psychiatry.2006;14:900-10.

54-O'Sullivan SB, Schmitz TJ. Fisioterapia avaliação e tratamento. $4^{\text {a }}$ Ed. Baาrueri: Manole, 2004, p.540.

55-Teixeira INDAO. O envelhecimento cortical e a reorganização neural após o acidente vascular encefálico (AVE): implicações para a reabilitação. Cienc Saúde Col 2008;13(supl.2):2171-8.

56-Ekman LL. Neurociência - Fundamentos para a reabilitação. São Paulo: Guanabara Koogan, 2000, p.153-7.

57-Fontes SV, Fukujima MM. Recuperação da Motricidade. In: Levy JA, Oliาveira ASB (eds). Reabilitação em doenças neurológicas: Guia terapêutico prátiาco. São Paulo: Atheneu, 2003, p.65-72. 